Received: 31 May 2018

Accepted: 14 January 2019

Published online: 06 March 2019

\section{Mouse mast cell protease 4 suppresses scar formation after traumatic spinal cord injury}

Tim Vangansewinkel ${ }^{1}$, Stefanie Lemmens ${ }^{1}$, Nathalie Geurts ${ }^{1}$, Kirsten Quanten ${ }^{1}$, Dearbhaile Dooley ${ }^{2}$, Gunnar Pejler ${ }^{3,4}$ \& Sven Hendrix $\mathbb{D}^{1}$

Spinal cord injury (SCl) triggers the formation of a glial and fibrotic scar, which creates a major barrier for neuroregenerative processes. Previous findings indicate that mast cells (MCs) protect the spinal cord after mechanical damage by suppressing detrimental inflammatory processes via mouse mast cell protease 4 (mMCP4), a MC-specific chymase. In addition to these immunomodulatory properties, mMCP4 also plays an important role in tissue remodeling and extracellular matrix degradation. Therefore, we have investigated the effects of $\mathrm{mMCP} 4$ on the scarring response after $\mathrm{SCl}$. We demonstrate that the decrease in locomotor performance in $\mathrm{mMCP4}^{-1-}$ mice is correlated with excessive scar formation at the lesion. The expression of axon-growth inhibitory chondroitin sulfate proteoglycans was dramatically increased in the perilesional area in $\mathrm{mMCP}^{-l-}$ mice compared to wild type mice. Moreover, the fibronectin-, laminin-, and collagen IV-positive scar was significantly enlarged in $\mathrm{mMCP}^{-I-}$ mice at the lesion center. A degradation assay revealed that $\mathrm{mMCP} 4$ directly cleaves collagen IV in vitro. On the gene expression level, neurocan and GFAP were significantly higher in the $\mathrm{mMCP4}^{-1-}$ group at day 2 and day 28 after injury respectively. In contrast, the expression of fibronectin and collagen IV was reduced in $\mathrm{MMCP}^{-1-}$ mice compared to WT mice at day 7 after SCI. In conclusion, our data show that mMCP4 modulates scar development after SCI by altering the gene and protein expression patterns of key scar factors in vivo. Therefore, we suggest a new mechanism via which endogenous $\mathrm{mMCP} 4$ can improve recovery after $\mathrm{SCl}$.

Spinal cord injury (SCI) is a chronic disorder that not only results in functional impairments and loss of sensation below the lesion site, but can also cause neuropathic pain and incontinence ${ }^{1,2}$. Currently, there are no effective therapies capable of restoring lost functions after injury. A major impediment for regenerative processes after SCI can be attributed to the expression of inhibitory factors that are associated with the lesion scar ${ }^{3,4}$. The scarring response is an evolving process, which involves various cells that accumulate at the lesion site (e.g. astrocytes, oligodendrocyte precursors, pericytes, ependymal cells) at different time points after injury ${ }^{5-10}$. These cellular responses result in the local deposition of extracellular matrix (ECM) components that form a dense scar. This scar is characterized by the expression of axon-growth inhibitory chondroitin sulfate proteoglycans (CSPGs) in the perilesional area ${ }^{11,12}$ and the formation of a collagenous and basement membrane-rich matrix in the lesion center, contributes to a physical barrier ${ }^{6,13,14}$. Although scar tissue displays favorable effects in the acute phase after SCI by restoring the tissue integrity and limiting secondary tissue damage ${ }^{6,15}$, it also blocks axon regeneration and other regenerative processes at later stages. Scar remodeling therapies are therefore of great interest in the SCI research field.

Mast cells (MCs) are immune cells characterized by electron-dense granules in their cytoplasm within which preformed mediators are stored, including cytokines and several MC-specific proteases (i.e. chymase and tryptase $)^{16-20}$. MCs reside in virtually all organs, including the brain and spinal cord ${ }^{21,22}$. As effector cells of the innate immune system, MCs from the periphery can also infiltrate the central nervous system (CNS) through a compromised blood brain barrier (BBB) which is characteristic of many neuroinflammatory diseases

${ }^{1}$ Department of Morphology, Biomedical Research Institute, Hasselt University, Diepenbeek, Belgium. ${ }^{2}$ Health Science Centre, School of Medicine, University College Dublin, Dublin, Ireland. ${ }^{3}$ Department of Anatomy, Physiology and Biochemistry, Swedish University of Agricultural Sciences, Uppsala, Sweden. ${ }^{4}$ Department of Medical Biochemistry and Microbiology, Uppsala University, Uppsala, Sweden. Tim Vangansewinkel and Stefanie Lemmens contributed equally. Correspondence and requests for materials should be addressed to S.H. (email: sven.hendrix@ uhasselt.be) 
and traumatic injuries ${ }^{23-25}$. As reviewed in Nelissen et al. ${ }^{26}$, MCs and their secreted mediators can modulate the inflammatory processes in multiple CNS pathologies. Amongst their complex effects, they can either contribute to neurological damage or provide neuroprotection. We have previously provided strong evidence demonstrating that MCs exert beneficial effects after traumatic CNS injury. Experiments in knockout mice indicated that MCs support neuronal survival and functional recovery after traumatic CNS injuries ${ }^{27,28}$. In particular, the protective effects of MCs appeared to be attributed due to their ability to promote the degradation of inflammation-associated cytokines such as interleukin 6 (IL-6) and monocyte chemoattractant protein-1 (MCP$1)$, thereby tempering 'detrimental' inflammatory processes. These immunomodulatory effects were partly mediated via mouse mast cell protease $4(\mathrm{mMCP} 4)^{28}$. This protease is the murine homolog of human $\alpha$-chymase $\mathrm{e}^{29}$; and it is a serine protease with chymotrypsin-like cleavage specificity ${ }^{30,31}$. A similar role for mMCP4 in the early inflammatory phase of experimental autoimmune encephalomyelitis (a mouse model of multiple sclerosis) has been reported, indicating immunomodulatory capacities also in other neurodegenerative diseases ${ }^{32}$.

Apart from its effects on the inflammatory response, mMCP4 is involved in ECM remodeling through direct cleavage of ECM components or, indirectly, by activating other ECM-processing enzymes (e.g. matrix metalloproteinases) ${ }^{16,33}$. By taking the ECM-degrading properties of mMCP4 into consideration, in this study, we investigated whether mMCP4 improves recovery after SCI by targeting the inhibitory lesion scar. We demonstrate that the absence of mMCP4 results in exacerbated scarring levels at the lesion site, suggesting an additional modulation effect of $\mathrm{mMCP} 4$. Hence, these data introduce a new potential mechanism via which MC chymase can alter the scar environment and support functional recovery after traumatic SCI.

\section{Results}

Impaired locomotor performance and increased scar formation in mMCP4 knockout mice after SCl. To determine the effect of $\mathrm{mMCP} 4$ on scar formation after SCI, we performed an in vivo experiment in which mMCP4 ${ }^{-1-}$ mice and their corresponding wild type (WT) controls were subjected to a dorsal T-cut hemisection lesion as described in the Methods section. A significant decrease in hind limb locomotor functions was observed in mMCP4 knockout mice compared to WT mice at 28 days post injury (dpi) (Fig. 1B). Histological analysis revealed that the fibrotic scar area (i.e. the GFAP negative area marked in green in Fig. 1Ai,ii) was significantly increased in $\mathrm{mMCP} 4^{-1-}$ mice compared to WT control mice (Fig. 1Ai/ii,C). We also observed an inverse correlation between fibrotic scar formation and the functional outcome after SCI in our mouse model $(\mathrm{p}=0.0456, \mathrm{r}=-0.4764$, Spearman rank correlation coefficient) (Fig. 1D). To analyze the fibrotic scar in more detail, we measured the area and immunoreactivity of key scar components at the lesion site, namely fibronectin, laminin and collagen type IV. We found that the fibronectin- (Fig. 1Aiii/iv,E), laminin- (Fig. 1Av/vi,H) and collagen IV-positive areas (Fig. 1Avii/viii,K) were significantly increased in $\mathrm{mMCP}^{-l-}$ compared to WT mice at $28 \mathrm{dpi}$. Moreover, also a significant inverse correlation was observed between the laminin-positive area and the functional outcome $(p=0.0343, r=-0.536)$ (Fig. 1I), but not with the other matrix components Fig. 1F,L). The intensity of staining for these scar components (within the positive area) has been analyzed as well and was comparable between $\mathrm{mMCP}^{-1-}$ and WT mice (Fig. 1A,G,J,M). Lastly, we analyzed the expression of axon-growth inhibitory CSPGs in a specifically-defined area around the lesion center (white encircled area in Fig. 2Aii/iv). Immunoreactivity for CSPGs was significantly higher in the perilesional area in $\mathrm{mMCP} 4^{-1-}$ mice compared to WT mice at 28 dpi (Fig. 2Aii/iv,B). Moreover, we also found a correlation between an increase of CSPG expression in the perilesional area and functional impairment after SCI, indicating an important role of CSPGs in SCI pathology $(\mathrm{p}=0.0135, \mathrm{r}=-0.57)$ (Fig. 2C).

Selected scar-associated ECM components are cleaved by mMCP4 in vitro. An in vitro degradation assay was performed to determine which scar-associated ECM components present as substrates for mMCP4. Recombinant fibronectin, laminin, collagen IV or a mix of CSPGs (aggrecan, neurocan, phosphacan, versican) were incubated with MC degranulate collected from either WT mice or $\mathrm{mMCP} 4^{-1-}$ mice. Cleavage fragments were visible after incubation with degranulate from both WT and $\mathrm{mMCP} 4^{-1-} \mathrm{MCs}$ (red-boxed areas in Fig. 3A), although quantification did not reveal any statistically significant effect of mMCP4-deficiency on the extent of CSPG degradation. This indicates that mMCP4 does not directly cleave CSPGs in our in vitro model. The protein band of fibronectin $(262 \mathrm{kDa})$ shows a decrease in intensity after incubation with degranulate from WT MCs (green-boxed area, Fig. 3B), and also cleavage products were observed at lower molecular weight levels (red-boxed area, Fig. 3B). When incubated with degranulate from mMCP4 ${ }^{-1-} \mathrm{MCs}$, considerably less degradation of fibronectin was visible (Fig. 3B). However, the differences in degradation between degranulate from WT MCs and $\mathrm{mMCP} 4^{-1-}$ MCs were not statistically significant. In addition, collagen type IV was cleaved after incubation with degranulate from WT MCs, as shown by a decrease in the intensity of the $250 \mathrm{kDa}$ protein band that corresponding to collagen IV (blue-boxed area, Fig. 3C). This effect was significantly reduced when collagen IV was incubated with degranulate from $\mathrm{mMCP} 4^{-1-} \mathrm{MCs}$, indicating that $\mathrm{mMCP} 4$ directly cleaves collagen type IV. Laminin is a trimeric protein with a molecular weight of $\sim 800 \mathrm{kDa}$ that consists of an $\alpha$-chain $(400 \mathrm{kDa})$, a $\beta$-chain $(200 \mathrm{kDa})$ and a $\gamma$-chain $(200 \mathrm{kDa})$, which are visible as two protein bands on the blot (Fig. 3D). Laminin was cleaved after incubation with degranulate from both WT and mMCP4 $4^{-1-}$ MCs (red-boxed area in Fig. 3D indicates cleavage fragments) and no difference was observed between the groups, indicating that mMCP4 does not directly cleave laminin in vitro (Fig. 3D).

Altered gene expression of scar-associated factors in MMCP4 knockout mice after SCl. Next, we addressed the question of whether endogenous mMCP4 influences the gene expression of important scar-associated markers after SCI. Quantitative PCR analysis showed that GFAP mRNA levels increased slightly after injury in both WT and $\mathrm{mMCP}^{-1-}$ mice (Fig. $4 \mathrm{~A}$ ). At $28 \mathrm{dpi}$, the expression level was higher in the $\mathrm{mMCP}^{-1-}$ vs. WT mice (fold change in expression vs. WT control condition: 3.590-fold vs. 1.786-fold) (Fig. 4A). 


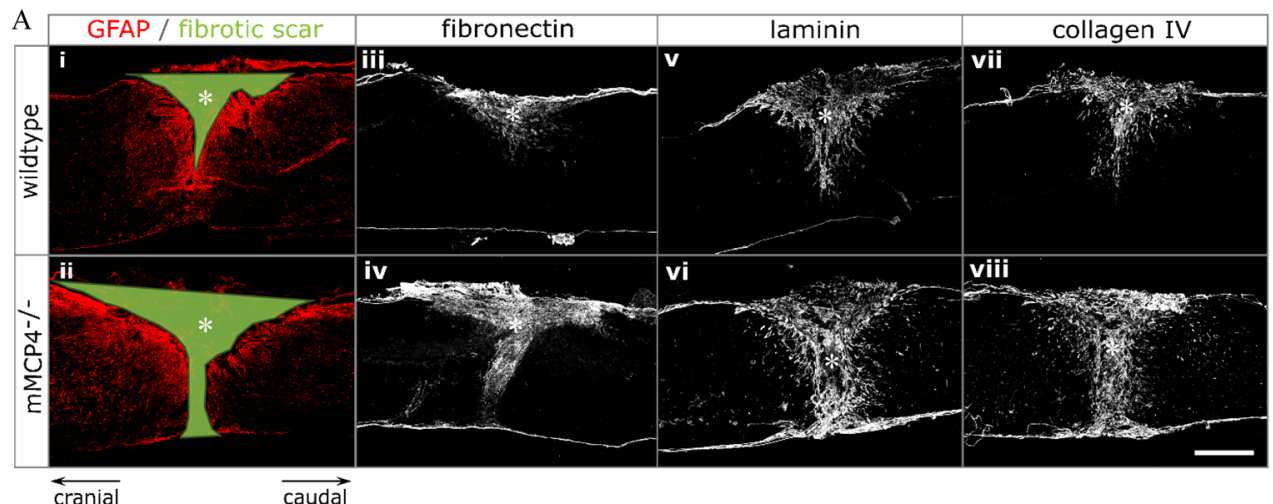

B

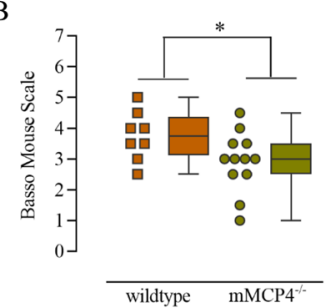

E

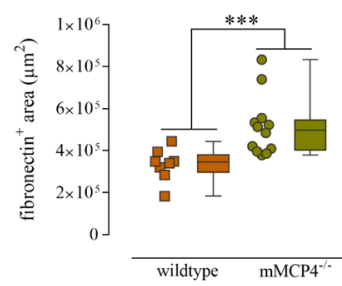

H

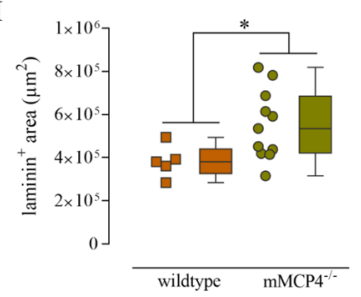

K

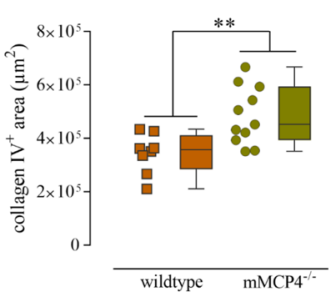

C

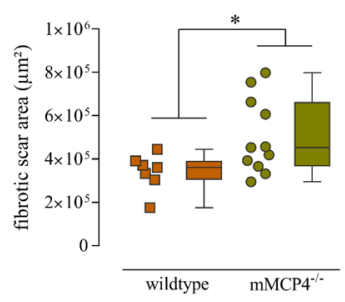

F
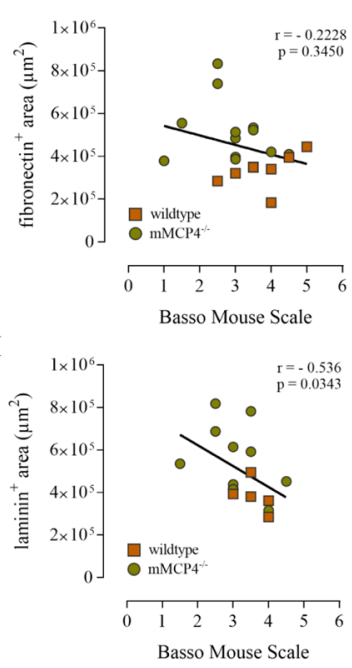

L

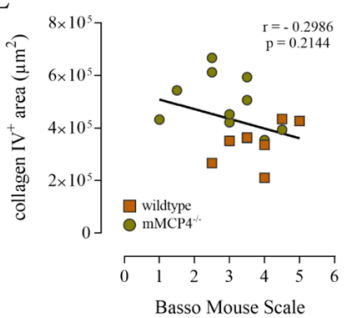

D

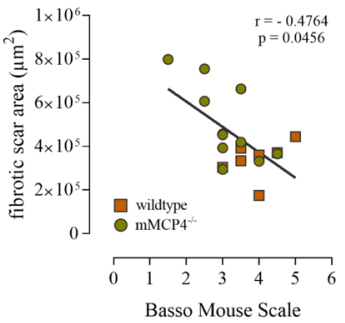

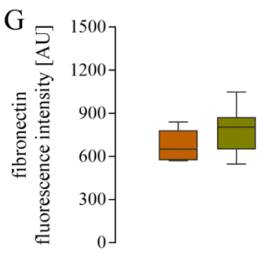

wildtype mMCP4

J
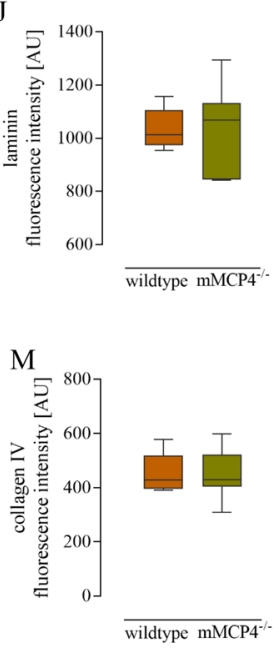

Figure 1. Impaired functional recovery and increased fibrotic scar formation in mMCP4 knockout mice after SCI. (A) Representative fluorescent photomicrographs of the fibrotic scar at the lesion site in WT and $\mathrm{mMCP} 4^{-1-}$ mice, respectively. Scale bar in all images $=500 \mu \mathrm{m}$. GFAP is visualized in red in Ai/ii. (B) The deficiency of mMCP4 in mMCP4 knockout mice results in a significantly decreased BMS score after SCI. WT mice: $\mathrm{n}=8 ; \mathrm{mMCP}^{-1-}$ mice: $\mathrm{n}=12$. (C) A significant increase in fibrotic scar area (i.e. GFAP negative area marked in green in Ai/ii) was observed in mMCP4 knockout mice (Aii) compared to WT controls (Ai) at 28 dpi. (D) Inverse correlation between fibrotic scar formation and functional outcome after SCI in our mouse model ( $\mathrm{p}=0.0456, \mathrm{r}=-0.4764$, Spearman rank correlation coefficient). ( $\mathbf{E}, \mathbf{H}, \mathbf{K})$ To characterize the fibrotic scar response in more detail, we examined the expression of fibronectin and of the basement membrane components laminin and collagen IV. We found that the fibronectin- (E), laminin- $(\mathbf{H})$ and collagen IV-positive (K) area were significantly increased in $\mathrm{mMCP}^{-1-}$ mice (Aiv/vi/viii) compared to WT mice (Aiii/v/vii). (F,I,L) A significant inverse correlation was observed between the laminin-positive area and the functional 
outcome ( $\mathrm{p}=0.0343, \mathrm{r}=-0.536)(\mathbf{I})$, but not with the other matrix components $(\mathbf{F}, \mathbf{L})$. $(\mathbf{G}, \mathbf{J}, \mathbf{M})$ In contrast to the area, the intensity of the immunoreactivity of these extracellular matrix components at the lesion was comparable between $\mathrm{WT}$ and $\mathrm{mMCP} 4^{-1-}$ mice. Individual data points are shown per mouse, together with the corresponding boxplots with the median and whiskers indicating the minimum and maximum. Histological analyses were performed on 5-8 WT mice and 10-12 $\mathrm{mMCP}^{-1-}$ mice. AU: arbitrary units. Asterisks in the fluorescent images indicate the lesion center. $* \mathrm{p}<0.05 ; * \mathrm{p}<0.01 ; * * \mathrm{p}<0.001$.

For the fibrous ECM component fibronectin, the mRNA levels were decreased at $7 \mathrm{dpi}$ in the $\mathrm{mMCP} 4^{-1-}$ mice (5.601-fold) compared with WT mice (27.192-fold) (Fig. 4B). Similarly, the expression levels of the basal lamina component collagen IV was decreased $7 \mathrm{dpi}$ in $\mathrm{mMCP} 4^{-1-}$ mice compared to the WT group (Fig. 4C). We also determined the gene expression profile of selected CSPGs, namely aggrecan, neurocan, and brevican (Fig. 4D-F). The aggrecan mRNA levels decreased at $2 \mathrm{dpi}$, increased at $7 \mathrm{dpi}$, after which they decreased again towards baseline levels. No differences were observed between WT and mMCP4 ${ }^{-1-}$ mice (Fig. 4D). Similarly to aggrecan, brevican mRNA levels were decreased $2 \mathrm{dpi}$ and no differences were observed between the experimental groups (Fig. 4E). Finally, neurocan mRNA levels were higher in the $\mathrm{mMCP}^{-1-}$ mice $(2.069$-fold) compared with WT mice (1.382-fold) at 2 dpi (Fig. $4 \mathrm{~F})$.

\section{Discussion}

Previously we have shown that MCs play a beneficial role in SCI pathology by reducing scar formation via $\mathrm{mMCP}^{34}$ and by suppressing detrimental inflammatory processes via mMCP4 $4^{27,28}$. In addition to its immunomodulatory properties, mMCP4 also plays a key role in tissue remodeling and ECM degradation via its own proteolytic capacities or via cleavage-activation of other proteolytic enzymes ${ }^{16,35}$. In this study we show for the first time that mMCP4 targets the inhibitory lesional scar after CNS injury. Similar to our findings in MC-deficient mice, we found that the decline in hind limb motor function in mMCP4 knockout mice (as reported in Nelissen et al. ${ }^{28}$ ) was associated with exacerbated scar formation, i.e. elevated expression of axon-growth inhibitory CSPGs in the perilesional area and increased deposition of the fibrotic scar components fibronectin, laminin and collagen IV in the lesion center after SCI. Moreover, we demonstrated that the elevated CSPG levels in the perilesional area and the increased fibrotic scar area were significantly correlated to the impaired motor performance in our mouse model (Figs 1D, I and 2B), highlighting the key role that these factors play in SCI pathology. Several studies have demonstrated that the inhibition of fibrotic scar components and CSPGs promotes regeneration of injured axons and improves functional recovery after CNS injury ${ }^{36-40}$. In a similar way, mMCP4 may degrade ECM components and, thereby, reduce scar tissue formation leading to better motor function. It is tempting to speculate that the larger fibrotic scar in mMCP4 knockout mice is a result of reduced scar compaction due to insufficient degradation of fibronectin, laminin and collagen IV. Increased scar compaction has been positively associated with better functional recovery after $\mathrm{SCI}^{41}$. This may in part explain decreased functional recovery in the knockout mice which show reduced scar compaction. Our in vivo findings are the first indication that mMCP4 suppresses scarring in the context of CNS trauma. Since scarring has an unfavorable impact on axon regeneration and other repair processes, it is tempting to speculate that the impaired functional outcome in mMCP4 knockout mice may be - at least in part - related to the increased scar formation at the lesion site.

Several studies have revealed that $\mathrm{mMCP} 4$ plays a crucial role in tissue remodeling and matrix degradation, both under physiological and pathological conditions ${ }^{16,33,35}$. Chymase/mMCP4 is a chymotrypsin-like protease that has a broad spectrum of activities against various ECM components. For example, it has been shown that $\mathrm{mMCP} 4$ has the ability to directly degrade fibronectin, and to indirectly influence ECM remodeling by activating pro-matrix metalloproteinase-2 (pro-MMP2) and pro-MMP9 ${ }^{31,42,43}$. In addition, it activates other pro-MMPs such as pro-MMP-1 and pro-MMP-3 that have potent matrix degrading properties ${ }^{44-46}$. Moreover, chymase can inactivate tissue inhibitor of matrix metalloproteinases (TIMPs), thereby increasing MMP activity and matrix remodeling ${ }^{47}$. Our degradation assays, performed with degranulate from WT and mMCP4 ${ }^{-1-} \mathrm{MCs}$, showed that collagen IV is a substrate of mMCP4. However, CSPGs and laminin were not cleaved in vitro. This finding suggests that additional mechanisms are involved in vivo via which $\mathrm{mMCP} 4$ reduces scarring after SCI, for example by activating pro-MMPs which in turn cleave ECM components of the fibrotic scars.

In addition, the immunomodulatory effects of $\mathrm{mMCP} 4$ may indirectly suppress the scarring response after CNS injury. Previously, we found that mMCP4 displays immunomodulatory functions ${ }^{28}$ by cleaving pro-inflammatory mediators that have detrimental effects after CNS injury. We have demonstrated that IL-6 is upregulated after SCI in mMCP4-deficient mice and is cleaved by mMCP4 ${ }^{28}$. Interestingly, IL-6 is a trigger of astrogliosis after $\mathrm{SCI}^{48,49}$ and these reactive astrocytes are also the main source of CSPGs produced at the lesion site after injury. Thus, the lack of mMCP4 in our mouse model may increase the deposition of CSPGs via increased IL- 6 or other pro-inflammatory cytokines. These findings are in line with increasing evidence suggesting that there is a strong interplay between the immune system and the ECM after CNS injury ${ }^{50,51}$. On the one hand, the glial scar contributes to protection of the spared neural tissues by establishing a boundary between damaged and healthy tissue, and by modulating the immune cells to promote the healing of the CNS tissue ${ }^{50,51}$. On the other hand, reduced fibrotic scar formation can lead to a decreased expression of tumor necrosis factor alpha ${ }^{52}$, which suggests that modulation of the fibrotic scar can also regulate the inflammatory response. Therefore we speculate that mMCP4 may modulate the interplay between the immune response and the scarring response in two ways: $\mathrm{mMCP} 4$ may reduce scarring following SCI by modulating inflammatory mediators $^{27,28}$ and it may suppress detrimental inflammatory processes in the injured CNS by cleaving and modifying scar-associated factors ${ }^{53,54}$. 

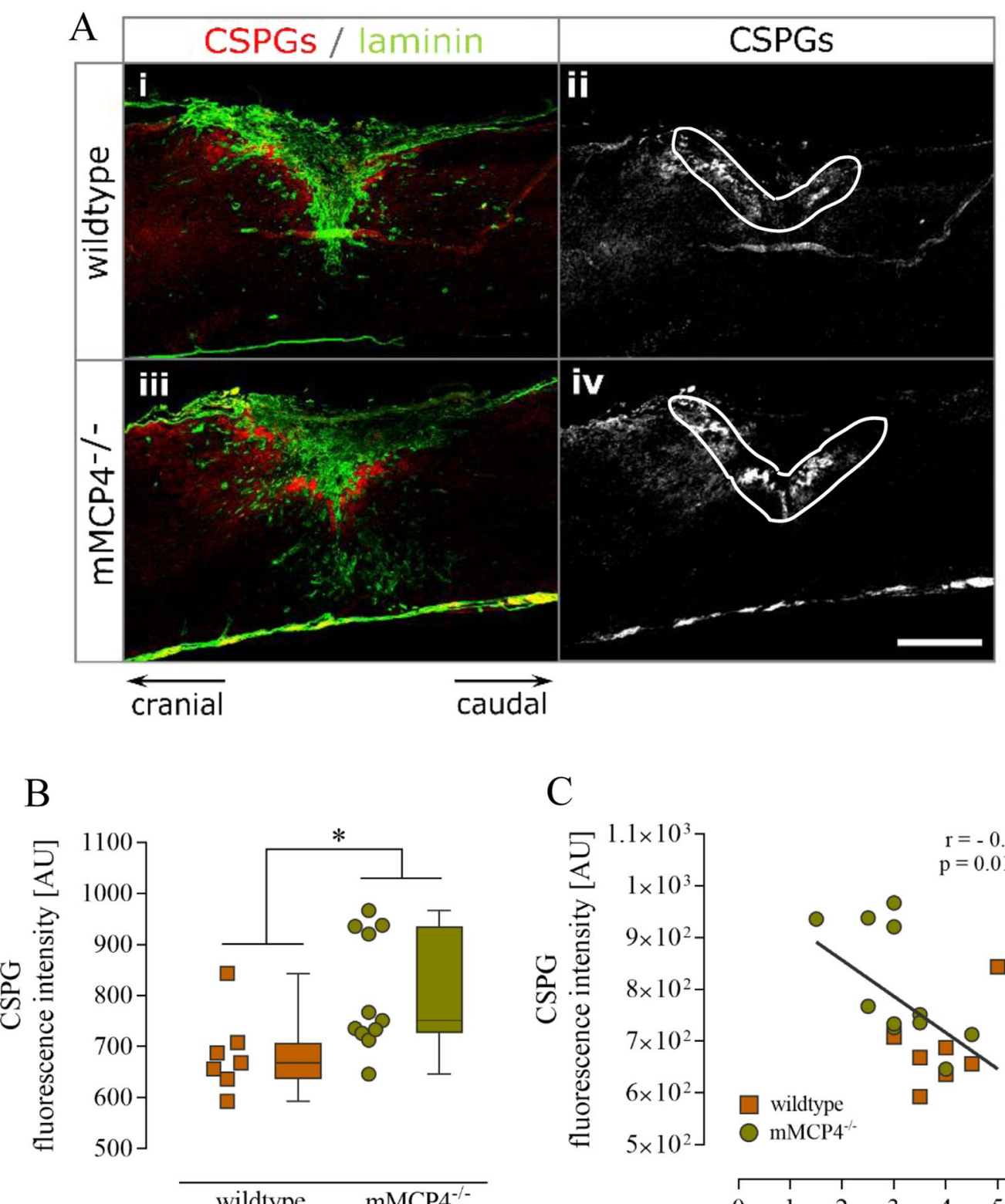

$\mathrm{C}$

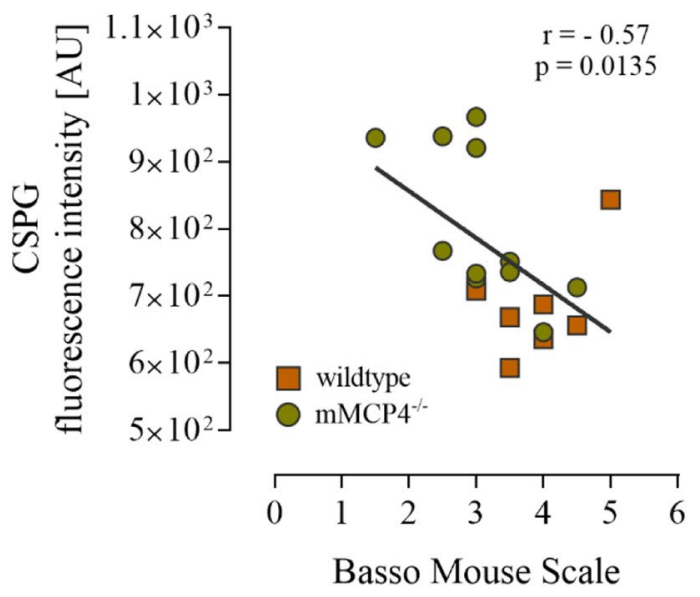

Figure 2. Increased expression of chondroitin sulfate proteoglycans in mMCP4 knockout mice after SCI. (A) Representative fluorescent photomicrographs of CSPG expression at the lesion site in WT and $\mathrm{mMCP} 4^{-1-}$ mice, respectively. Scale bars $=500 \mu \mathrm{m}$. Immunofluorescence for laminin (green) was performed to highlight the lesion center, and CSPGs (red) are strongly upregulated in the perilesional area after traumatic SCI (Ai/iii). (B) A significant increase in CSPG immunoreactivity was observed in the perilesional area in $\mathrm{mMCP}^{-1-}$ (Aiii/iv) compared to WT mice (Ai/ii) (area encircled by white line in Aii/iv indicates the analyzed region). (C) Inverse correlation between CSPG expression at the lesion site and the functional outcome after SCI in our mouse model $(\mathrm{p}=0.0135, \mathrm{r}=-0.57$, Spearman rank correlation coefficient). Individual data points are shown per mouse, together with the corresponding boxplots with the median and whiskers indicating the minimum and maximum. WT mice: $\mathrm{n}=7 ; \mathrm{mMCP} 4^{-/-}$mice: $\mathrm{n}=11$. AU: arbitrary units. Asterisks in the images indicate the lesion center. $* \mathrm{p}<0.05$

Finally, we investigated whether the absence of mMCP4 affected the expression of selected genes linked to scarring after SCI. We observed increased GFAP mRNA expression levels in $\mathrm{mMCP} 4^{-1-}$ mice at 28 dpi. Interestingly, the difference in GFAP expression between $\mathrm{mMCP} 4^{-1-}$ and WT mice in the perilesional area is not reflected at the protein level ${ }^{28}$. In addition, the mRNA levels of the CSPG neurocan were elevated at 2 dpi in the $\mathrm{mMCP}^{-1-}$ mice compared to controls. Surprisingly, in the absence of mMCP4, the gene expression of fibronectin and collagen IV remained low compared to the significantly elevated mRNA levels in WT mice at $7 \mathrm{dpi}$. In contrast, at the protein level the fibronectin-positive and collagen IV-positive areas were significantly increased 
A

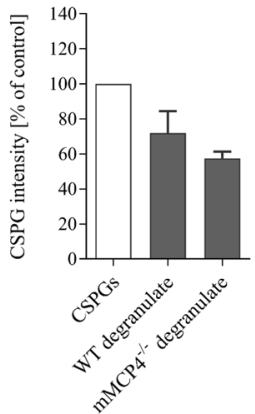

$\mathrm{C}$

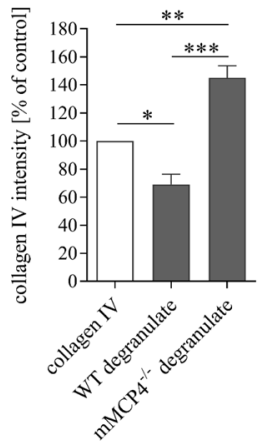

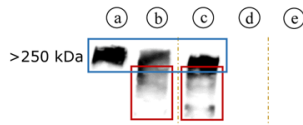

Collagen IV

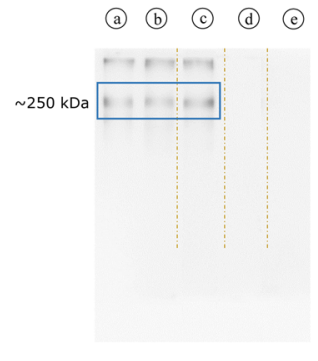

$\mathrm{B}$

Fibronectin

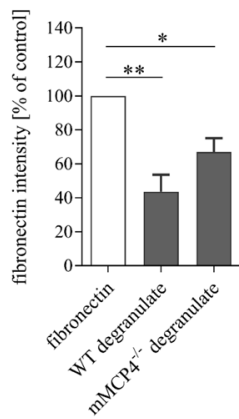

(a) (b) (c) (d) (c)

$\sim 262 \mathrm{kDa}$

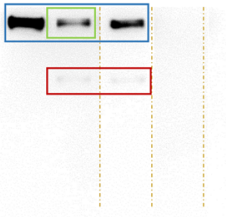

$\mathrm{D}$

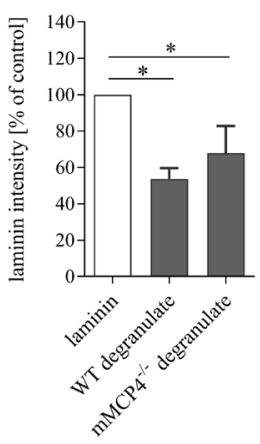

Figure 3. $\mathrm{mMCP} 4$ degrades scar-associated ECM components in vitro. (A-D) Recombinant fibronectin, collagen IV, laminin or a CSPG-mix (aggrecan, neurocan, phosphacan, versican) were incubated with degranulate from WT or mMCP4 ${ }^{-1-}$ MCs to measure protein degradation. (A) Fragments of CSPG degradation products are visible at lower molecular weight levels after incubation with degranulate from WT or $\mathrm{mMCP} 4^{-1-}$ MCs (red-boxed areas in A). No statistically significant difference in CSPG intensity was observed between the groups. (B) Fibronectin was cleaved by degranulate from both WT and $\mathrm{mMCP} 4^{-1-} \mathrm{MCs}$ (the red-boxed area indicates cleavage fragments); although with reduced cleavage (trend) by degranulate from $\mathrm{mMCP} 4^{-1-}$ vs. WT MCs (the green box indicates a stronger reduction in intensity of the fibronectin protein band after incubation with $\mathrm{MC}_{\mathrm{WT}}$ degranulate). (C) Collagen IV was cleaved by degranulate from WT MCs, but not degranulate from mMCP4 $4^{-1-}$ MCs. (D) Laminin was cleaved by degranulate from both WT and $\mathrm{mMCP} 4^{-1-} \mathrm{MCs}$ (the red box indicates cleavage fragments). Data were normalized to the control condition (a) and presented as mean $\pm \mathrm{SEM}$; $\mathrm{n}=3-4$ experimental repeats/condition; ${ }^{\mathrm{p}} \mathrm{p}<0.05$, ** $\mathrm{p}<0.01, * * * \mathrm{p}<0.001$. Legend: $(\mathrm{a})=$ recombinant protein; $(\mathrm{b})=$ recombinant protein $+\mathrm{MC}_{\mathrm{WT}}$ degranulate; (c) = recombinant protein $+\mathrm{MC}_{\mathrm{mMCP} 4}{ }^{-1-}$ degranulate; (d) = degranulate from WT MCs alone; (e) degranulate from $\mathrm{mMCP}^{-1-} \mathrm{MCs}$ alone. Intensity analysis were performed on the main protein bands that correspond with the known molecular weights of the ECM components (blue boxes). Brown dotted lines in the images indicate that the original blots have been cropped to exclude data on mMCP6 degradation which are not the focus of this study and have been published in Vangansewinkel et al. ${ }^{34}$. Original uncropped Western blot images are shown in Supplementary Fig. S1.

in the $\mathrm{mMCP} 4^{-1-}$ group. It is important to note that the increased levels of fibronectin and collagen IV mRNA are detected early ( $7 \mathrm{dpi}$ ), while the fibronectin and collagen IV protein increases are found at $28 \mathrm{dpi}$. Moreover, the mRNA data for fibronectin, collagen IV, aggrecan, brevican and neurocan indicate no substantial change at 28 dpi suggesting that the observed differences between $\mathrm{mMCP} 4^{-1-}$ mice and WT mice on the protein level are the result of degradation, and not of regulatory effects on the gene level or of protein production.

To conclude, in this study, we demonstrate that the absence of mMCP4 in knockout mice results in exacerbated scar formation and this correlated with a reduction in functional recovery after SCI. These data reveal a new mechanism in which endogenous MMCP4 may support recovery after CNS injury via scar remodeling - in addition to its immunomodulatory properties which we have demonstrated previously ${ }^{28}$. Future research will reveal whether therapeutic administration of recombinant $\mathrm{mMCP} 4$ improves functional regeneration via scar remodeling and/or modulation of the immune system.

\section{Methods}

Animals and spinal cord injury. We used mMCP4 knockout mice (mMCP4 ${ }^{-1-} ; 10-12$ weeks old), which were backcrossed for at least 10 -generations to a C57BL/6 background ${ }^{31}$. WT C57BL/6j mice (Janvier) of the same age were used as controls. All mice were housed in a conventional animal facility at Hasselt University under regular conditions, i.e. in a temperature-controlled room $\left(20 \pm 3^{\circ} \mathrm{C}\right)$ on a $12 \mathrm{~h}$ light-dark schedule and with food and 
A

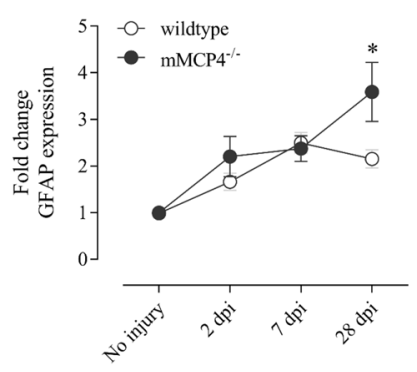

$\mathrm{D}$

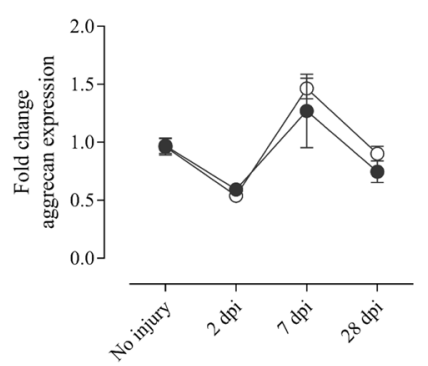

B

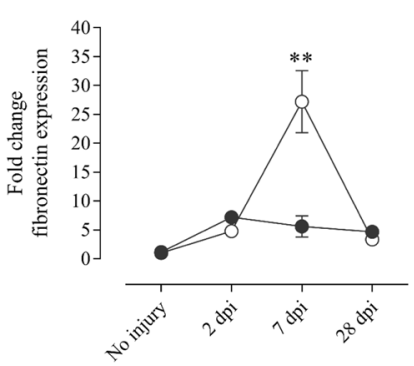

E

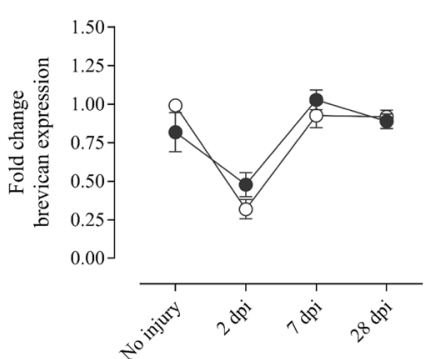

$\mathrm{C}$

Collagen IV

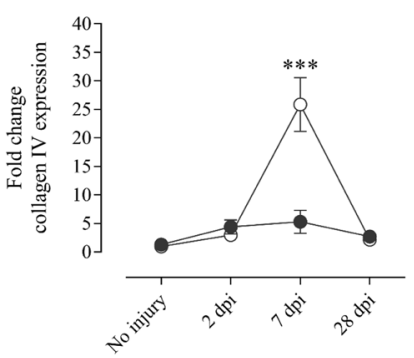

$\mathrm{F}$

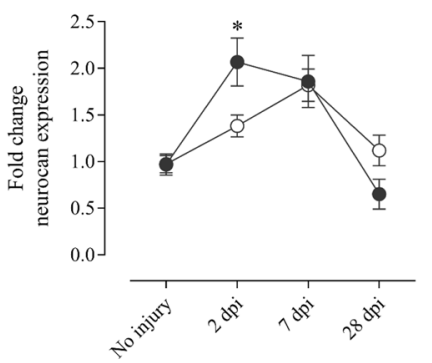

Figure 4. Altered expression of genes coding for scar-associated components after SCI. (A-F) mRNA expression levels of GFAP, fibronectin, collagen IV, aggrecan, brevican, and neurocan were measured by quantitative PCR analysis in spinal cord tissue from WT (white circles) and $\mathrm{mMCP}^{-1-}$ mice (black circles) at $2 \mathrm{dpi}, 7 \mathrm{dpi}$, and $28 \mathrm{dpi}$. Samples from mice that did not undergo surgery were included as a control. (A) GFAP mRNA levels were significantly elevated in $\mathrm{mMCP}^{-1-}$ mice compared with WT mice at $28 \mathrm{dpi}$. (B,C) In contrast, fibronectin and collagen IV gene expression were increased in the WT group compared to mMCP4 ${ }^{-1-}$ mice at 7 dpi. Out of the CSPGs, (D) aggrecan and (E) brevican mRNA were both not differentially expressed after injury whereas the expression of $(\mathbf{F})$ neurocan was increased in $\mathrm{mMCP}^{-1-}$ mice compared to the WT mice at 2 dpi. Expression levels were normalized to the reference genes YHWAZ and CYCA, and converted to fold change values vs. the WT control condition using the $2^{-\Delta \Delta C T}$ method as described in the methods. Data are presented as mean \pm SEM; $\mathrm{n}=5-9$ mice analyzed/group; ${ }^{*} \mathrm{p}<0.05, * * \mathrm{p}<0.01,{ }^{* * *} \mathrm{p}<0.001$.

water ad libitum. All experiments were approved by the local ethical committee of Hasselt University, and were performed according to the guidelines described in Directive 2010/63/EU.

A T-cut hemisection injury was performed as previously described ${ }^{34,55}$. Briefly, mice were anesthetized by inhalation anesthesia with $3 \%$ isoflurane (IsofFlo, Abbot Animal Health) and $0_{2}$ as a carrier gas; and the spinal cord was exposed by performing a partial laminectomy at thoracic level 8 (T8). Then a bilateral hemisection injury of the spinal cord was induced by using iridectomy scissors to transect the left and right dorsal funiculus, the dorsal horns and the ventral funiculus. Locomotor recovery of the animals at 28 dpi was determined by using the Basso Mouse Scale (BMS) ${ }^{56}$.

Histological analysis. At $28 \mathrm{dpi}$, mice received an overdose of Nembutal and they were transcardially perfused with Ringer solution containing heparin, followed by $4 \%$ paraformaldehyde in PBS (pH 7.4). Next, $14 \mu \mathrm{m}$ thick sagittal tissue sections were cut and immunohistochemical stainings were performed as previously described $^{34}$. Spinal cord sections were blocked with $10 \%$ normal goat serum and permeabilized with $0.05 \%$ Triton X-100 in PBS for $30 \mathrm{~min}$ at room temperature (RT). Then, the following primary antibodies were incubated overnight at $4{ }^{\circ} \mathrm{C}$ in a humidified chamber: monoclonal mouse anti-glial fibrillary acidic protein (GFAP) (1:500, G3893, Sigma-Aldrich), polyclonal rabbit anti-laminin 1+2 (1:200, ab7463, Abcam), polyclonal rabbit anti-fibronectin (1:200, ab2413, Abcam), polyclonal rabbit anti-collagen IV (1:200, ab6586, Abcam) and monoclonal mouse anti-CSPGs (1:200; CS-56; C8035, Sigma-Aldrich). The CS-56 antibody specifically detects the glycosaminoglycan (GAG) portion of native CSPG molecules (e.g. versican, brevican, neurocan). Following repeated washing steps with PBS, spinal cord sections were incubated with Alexa-labeled secondary antibodies for $1 \mathrm{~h}$ at RT, namely goat anti-mouse IgG Alexa 555, goat anti-rabbit Alexa 488 and goat anti-mouse IgM Alexa 555 ( $1: 250$, secondary antibodies were obtained from Invitrogen). A 4,6-diamino-2-phenylindole (DAPI, Invitrogen) counterstain was performed to reveal cellular nuclei and sections were mounted. Images were taken with a Nikon Eclipse 80i microscope equipped with a Nikon digital sight camera DS-2MBWc. Quantitative image analysis were performed on original unmodified photos using the ImageJ open source software (National Institutes of Health). For standardization, analyses were performed on 5-8 spinal cord sections (per mouse) representing the lesion area (i.e. the lesion epicenter as well as consecutive sagittal sections), as previously described ${ }^{34,57}$. The spinal cord 


\begin{tabular}{|l|l|l|l|l|}
\hline Components & Gene name & Accession number & Primer sequence 5' $\rightarrow 3^{\prime}$ & $\begin{array}{l}\text { Amplicon } \\
\text { length (bp) }\end{array}$ \\
\hline $\begin{array}{l}\text { Gliosis } \\
\text { GFAP }\end{array}$ & Gfap & NM_001131020.1 & $\begin{array}{l}\text { FW: TCTCCAACCTCCAGATCCGA } \\
\text { Rev: CTGGTGAGCCTGTATTGGGA }\end{array}$ & 113 \\
\hline $\begin{array}{l}\text { Scar components } \\
\text { Fibronectin 1 }\end{array}$ & Fn1 & NM_010233.1 & $\begin{array}{l}\text { FW: ATGTGGACCCCTCCTGATAGT } \\
\text { Rev: GCCCAGTGATTCAGCAAAGG }\end{array}$ & 124 \\
\hline Collagen, type IV, $\alpha$ I & Col4al & NM_009931.2 & $\begin{array}{l}\text { FW: AACAACGTCTGCAACTTCGC } \\
\text { Rev: CTTCACAAACCGCACACCTG }\end{array}$ & 136 \\
\hline Aggrecan & Acan & NM_007424.2 & $\begin{array}{l}\text { FW: GTCGCTCCCCAACTATCCAG } \\
\text { Rev: AAAGTCCAGGGTGTAGCGTG }\end{array}$ & 193 \\
\hline Neurocan & Ncan & X84727.1 & $\begin{array}{l}\text { FW: CACAGAAGTGAGATCAGTGAGA } \\
\text { Rev: GCACCATCTTGGTTCAGGCA }\end{array}$ & 114 \\
\hline Brevican & Bcan & X87096.1 & $\begin{array}{l}\text { FW: TGCCGAAGACCTAAATGGAGA } \\
\text { Rev: CACGTTCCAGACAGTAGTCCC }\end{array}$ & 89 \\
\hline $\begin{array}{l}\text { Reference genes } \\
\text { CYCA }\end{array}$ & Ccna2 & NM_009828.2 & $\begin{array}{l}\text { FW: GCGTCTCCTTCGAGCTGTT } \\
\text { Rev: AAGTCACCACCCTGGCA }\end{array}$ & 108 \\
\hline YWHAZ & Ywhaz & NM_011740.3 & $\begin{array}{l}\text { FW: CAACGATGTACTGTCTCTTTTGG } \\
\text { Rev: GTCCACAATTCCTTTCTTGTCATC }\end{array}$ & 149 \\
\hline
\end{tabular}

Table 1. Overview gene-specific primers used for quantitative PCR analysis. ${ }^{\mathrm{a}} \mathrm{NCBI}$ accession number of mRNA and corresponding gene, available at http://www.ncbi.nlm.nih.gov/gene GFAP: glial fibrillary acidic protein; CYCA: cyclin A2 YHWAZ: tyrosine 3-monooxygenase/tryptophan 5-monooxygenase activation protein zeta.

lesion microenvironment can in general be subdivided in different regions, namely the lesion center, the perilesional area and the surrounding tissue. Fibrotic scar tissue is deposited in the lesion center which is surrounded by GFAP-positive astrocytes (=the glial scar) ${ }^{5,6,38,58}$. Therefore, the fibrotic scar area (highlighted in green in Fig. $1 \mathrm{Ai} /$ ii) was evaluated by delineating the area in which there was no GFAP-immunoreactivity. To characterize the fibrotic scar in more detail, we also determined the area and immunoreactivity of the following matrix components at the lesion: fibronectin, laminin and collagen IV. To evaluate the expression of CSPGs, intensity analysis of CSPG-immunoreactivity was measured perilesionally in a well-defined area surrounding the lesion center ( $200 \mu \mathrm{m}$ zone surrounding the lesion center). Representative fluorescent photomicrographs are shown in Figs $1 \mathrm{~A}$ and $2 \mathrm{~A}$. To maximise image readability, the contrast and brightness of the stainings was enhanced equally for WT and $\mathrm{mMCP} 4^{-1-}$ mice.

Quantitative PCR analysis. mRNA expression levels of glial and fibrotic scar-associated components were investigated at different phases after SCI, namely the acute phase ( $2 \mathrm{dpi}$ ), the subacute phase ( $7 \mathrm{dpi}$ ), and finally the early stage of the chronic remodeling phase ( $28 \mathrm{dpi})$. At these selected time points after injury, WT C57BL/6 mice and $\mathrm{mMCP} 4^{-1-}$ mice were transcardially perfused with Ringer solution as described. Healthy mice (without SCI) were included as controls in the analysis. Standardized areas of spinal cord tissue $(5 \mathrm{~mm}$ cranial and $5 \mathrm{~mm}$ caudal to the lesion center) were collected and mRNA was extracted using the Paris Kit (Life Technologies), according to the manufacturer's instructions with minor modifications as described in Vangansewinkel et al. ${ }^{34}$. Reverse transcription to cDNA (VWR) was performed following the reaction protocol provided with the $\mathrm{qS}_{\mathrm{S}}$ (ript $^{\mathrm{TM}}$ cDNA SuperMix.

Quantitative PCR was conducted on a StepOnePlus detection system (Applied Biosystems) using universal cycling conditions $\left(20 \mathrm{~s}\right.$ at $95^{\circ} \mathrm{C}, 40$ cycles of $3 \mathrm{~s}$ at $95^{\circ} \mathrm{C}$ and $30 \mathrm{~s}$ at $60^{\circ} \mathrm{C}$ ). The reaction mixture contained fast SYBR Green master mix (Applied Biosystems), $10 \mathrm{mM}$ of forward and reverse primers (Eurogentec), RNase free water, and $8 \mathrm{ng}$ template cDNA in a total reaction volume of $10 \mu \mathrm{l}$. The primer sequences used are shown in Table 1. Relative quantification of gene expression was accomplished by using the $2^{-\Delta \Delta \mathrm{CT}}$ method and data were normalized to the most stable reference genes. Briefly, GeNorm software identified cyclophilin A (CYCA) and tyrosine 3-monooxygenase/tryptophan 5-monooxygenase activation protein $\mathrm{z}$ (YWHAZ) as the most stable reference genes. Allprimers were designed using Primer-Express (http://www.ncbi.nlm.nih.gov/tools/primer-blast). The gene expression levels are presented as fold change of the WT control condition.

Extracellular matrix degradation assays. Degradation assays were performed to determine which scar-associated ECM components are a substrate of mMCP4 in vitro. MC degranulate was obtained from WT and $\mathrm{mMCP}^{-1-}$ mice as previously described ${ }^{28,34}$. Murine recombinant fibronectin $(1 \mu \mathrm{g} ; \mathrm{Abcam})$, laminin $(0.5 \mu \mathrm{g}$; Millipore) or a CSPG-mix (CC117, $2 \mu$; Millipore) were incubated with $20 \mu \mathrm{l}$ MilliQ or with $20 \mu \mathrm{l}$ degranulate obtained from either WT or mMCP4 ${ }^{-I-} \mathrm{MCs}$ for $48 \mathrm{~h}$ at $37^{\circ} \mathrm{C}$. After incubation, samples were mixed with reducing sample buffer and the cleaved fragments were identified via SDS-PAGE and Western blot analysis. Briefly, sample buffer containing 5\% $\beta$-mercaptoethanol (Fluka Biochemika) was added and the incubation mixture was denaturated at $95^{\circ} \mathrm{C}$ for $5 \mathrm{~min}$. Western blotting for collagen IV was performed under non-reducing conditions (no denaturation with $\beta$-mercaptoethanol). Protein samples were separated on $7.5 \%$ polyacrylamide gels, containing Tris-glycine and transferred onto polyvinylidene fluoride membranes. Membranes were blocked in $5 \%$ nonfat powdered milk in tris-buffered saline-Tween $20(0.1 \%)$ (TBST) for $1 \mathrm{~h}$ and probed overnight at $4{ }^{\circ} \mathrm{C}$ with one of the following primary antibodies: polyclonal rabbit anti-fibronectin (1:1000, sc-9068, Santa Cruz), anti-collagen IV (1:1000, ab6586, Abcam), anti-laminin 1+2 (1:1000, ab7463, Abcam) and anti-CSPGs (1:1000, CS56, C8035, Sigma-Aldrich). Next, membranes were washed with TBST and incubated with the appropriate 
HRP-conjugated secondary antibodies: goat anti-mouse IgM and goat anti-rabbit (dilution 1:5000; all secondary antibodies were obtained from Dako). An ECL Plus detection kit (Thermo Scientific, Pierce ${ }^{\circledR}$ ) was used and the generated chemiluminescent signal was detected using a luminescent image analyzer (ImageQuant LAS 4000 mini). Quantitative analysis were performed on original unmodified blots and densitometry of the protein bands (corresponding to the known molecular weight of the respective ECM components - highlighted by blue boxes in the blots of Fig. 3) was quantified via the ImageQuant TL software. For laminin, the density of the two molecular weight bands were summed together (Fig. 3D). The density of the cleavage fragments has not been measured because we cannot guarantee that the used antibodies are able to detect all cleavage fragments because they are produced to target the native ECM protein. Therefore, a reduction in the density of the ECM component protein band suggests a reduction in the amount of protein, or it may indicate cleavage. The densities of the experimental conditions (containing degranulate from WT or $\mathrm{mMCP}^{-1-} \mathrm{MCs}$ ) have been normalized to the densities of unstimulated control bands. To minimize bias due to differences in densitometric measurements between experiments, each control condition per experiment was set at $100 \%$, thereby lacking a standard error bar. It is important to note that the ECM degradation assays were run in parallel with our previous study ${ }^{34}$ to guarantee comparability between the cleavage effects of mMCP6 and mMCP4 on scar components. This implicates that we used the same control groups for both studies. The control graphs in Fig. 3A-D, i.e. group a (recombinant protein) and group $\mathrm{b}$ (recombinant protein $+\mathrm{MC}_{\mathrm{WT}}$ degranulate) are reprinted with permission from The Faseb Journal. To improve readability of the images, the contrast and brightness was modified in the representative protein blots that are displayed in Fig. 3. Original Western blots are shown in Supplementary Fig. S1.

Statistical analysis. All statistical analyses were performed using GraphPad Prism 5.01 software (GraphPad Software, Inc.). Data sets were analyzed for normal distribution using the D'Agostino-Pearson normality test. Histological differences between WT and knockout mice at 28 dpi were statistically analyzed using the nonparametric Mann-Whitney U test. Individual data points are shown per mouse, and also the corresponding box plots with the median and whiskers indicating the minimum and maximum are presented (Figs 1 and 2). Correlative analysis between the functional outcome and scar formation (fibrotic scar area and CSPG expression) after SCI was performed with the Spearman rank correlation test. In vitro ECM degradation assays were analyzed with a one-way ANOVA to compare multiple groups followed by a Tukey post hoc test. Quantitative PCR data were analyzed using two-way ANOVA with a Bonferroni post hoc test. These data were presented as mean \pm standard error of the mean (Figs 3 and 4). At 95\% confidence interval, differences were considered statistically significant when $\mathrm{p}<0.05$.

\section{Data Availability}

All data supporting the findings of this study are available from the corresponding author on reasonable request.

\section{References}

1. Ahuja, C. S. et al. Traumatic spinal cord injury. Nat Rev Dis Primers 3, 17018, https://doi.org/10.1038/nrdp.2017.18 (2017).

2. Bradbury, E. J. \& McMahon, S. B. Spinal cord repair strategies: why do they work? Nat Rev Neurosci 7, 644-653, https://doi. org/10.1038/nrn1964 (2006).

3. Silver, J. \& Miller, J. H. Regeneration beyond the glial scar. Nat Rev Neurosci 5, 146-156, https://doi.org/10.1038/nrn1326 (2004).

4. Fawcett, J. W. \& Asher, R. A. The glial scar and central nervous system repair. Brain research bulletin 49, 377-391 (1999).

5. Soderblom, C. et al. Perivascular fibroblasts form the fibrotic scar after contusive spinal cord injury. The Journal of neuroscience: the official journal of the Society for Neuroscience 33, 13882-13887, https://doi.org/10.1523/JNEUROSCI.2524-13.2013 (2013).

6. Goritz, C. et al. A pericyte origin of spinal cord scar tissue. Science 333, 238-242, https://doi.org/10.1126/science.1203165 (2011).

7. Sofroniew, M. V. Molecular dissection of reactive astrogliosis and glial scar formation. Trends in neurosciences 32, 638-647, https:// doi.org/10.1016/j.tins.2009.08.002 (2009).

8. Ren, Y. et al. Ependymal cell contribution to scar formation after spinal cord injury is minimal, local and dependent on direct ependymal injury. Sci Rep 7, 41122, https://doi.org/10.1038/srep41122 (2017).

9. Dias, D. O. et al. Reducing Pericyte-Derived Scarring Promotes Recovery after Spinal Cord Injury. Cell 173, 153-165 e122, https:// doi.org/10.1016/j.cell.2018.02.004 (2018).

10. Li, X. et al. Regenerative Potential of Ependymal Cells for Spinal Cord Injuries Over Time. EBioMedicine 13, 55-65, https://doi. org/10.1016/j.ebiom.2016.10.035 (2016).

11. Jones, L. L., Sajed, D. \& Tuszynski, M. H. Axonal regeneration through regions of chondroitin sulfate proteoglycan deposition after spinal cord injury: a balance of permissiveness and inhibition. The Journal of neuroscience: the official journal of the Society for Neuroscience 23, 9276-9288 (2003).

12. Cregg, J. M. et al. Functional regeneration beyond the glial scar. Experimental neurology 253, 197-207, https://doi.org/10.1016/j. expneurol.2013.12.024 (2014).

13. Hermanns, S., Klapka, N., Gasis, M. \& Muller, H. W. The collagenous wound healing scar in the injured central nervous system inhibits axonal regeneration. Advances in experimental medicine and biology 557, 177-190, https://doi.org/10.1007/0-387-301283_11 (2006).

14. Moeendarbary, E. et al. The soft mechanical signature of glial scars in the central nervous system. Nat Commun 8, 14787, https://doi. org/10.1038/ncomms14787 (2017).

15. Rolls, A., Shechter, R. \& Schwartz, M. The bright side of the glial scar in CNS repair. Nat Rev Neurosci 10, 235-241, https://doi. org/10.1038/nrn2591 (2009).

16. Pejler, G., Ronnberg, E., Waern, I. \& Wernersson, S. Mast cell proteases: multifaceted regulators of inflammatory disease. Blood 115 , 4981-4990, https://doi.org/10.1182/blood-2010-01-257287 (2010).

17. Lundequist, A. \& Pejler, G. Biological implications of preformed mast cell mediators. Cellular and molecular life sciences: CMLS 68, 965-975, https://doi.org/10.1007/s00018-010-0587-0 (2011).

18. Wernersson, S. \& Pejler, G. Mast cell secretory granules: armed for battle. Nat Rev Immunol 14, 478-494, https://doi.org/10.1038/ nri3690 (2014).

19. Galli, S. J., Grimbaldeston, M. \& Tsai, M. Immunomodulatory mast cells: negative, as well as positive, regulators of immunity. Nat Rev Immunol 8, 478-486, https://doi.org/10.1038/nri2327 (2008).

20. Mukai, K., Tsai, M., Saito, H. \& Galli, S. J. Mast cells as sources of cytokines, chemokines, and growth factors. Immunol Rev 282, 121-150, https://doi.org/10.1111/imr.12634 (2018). 
21. Hendrix, S. et al. The majority of brain mast cells in B10.PL mice is present in the hippocampal formation. Neurosci Lett 392, 174-177 (2006).

22. Sayed, B. A., Christy, A. L., Walker, M. E. \& Brown, M. A. Meningeal mast cells affect early T cell central nervous system infiltration and blood-brain barrier integrity through TNF: a role for neutrophil recruitment? J Immunol 184, 6891-6900, https://doi. org/10.4049/jimmunol.1000126 (2010).

23. Bennett, J. L. et al. Bone marrow-derived mast cells accumulate in the central nervous system during inflammation but are dispensable for experimental autoimmune encephalomyelitis pathogenesis. J Immunol 182, 5507-5514, https://doi.org/10.4049/ jimmunol.0801485 (2009).

24. Lindsberg, P. J., Strbian, D. \& Karjalainen-Lindsberg, M. L. Mast cells as early responders in the regulation of acute blood-brain barrier changes after cerebral ischemia and hemorrhage. J Cereb Blood Flow Metab 30, 689-702, https://doi.org/10.1038/ jcbfm.2009.282 (2010).

25. Russi, A. E., Walker-Caulfield, M. E. \& Brown, M. A. Mast cell inflammasome activity in the meninges regulates EAE disease severity. Clin Immunol, https://doi.org/10.1016/j.clim.2016.04.009 (2016).

26. Nelissen, S. et al. The role of mast cells in neuroinflammation. Acta Neuropathol 125, 637-650, https://doi.org/10.1007/s00401-0131092-y (2013).

27. Hendrix, S. et al. Mast cells protect from post-traumatic brain inflammation by the mast cell-specific chymase mouse mast cell protease-4. FASEB journal: official publication of the Federation of American Societies for Experimental Biology 27, 920-929, https:// doi.org/10.1096/fi.12-204800 (2013).

28. Nelissen, S. et al. Mast cells protect from post-traumatic spinal cord damage in mice by degrading inflammation-associated cytokines via mouse mast cell protease 4. Neurobiology of disease, https://doi.org/10.1016/j.nbd.2013.09.012 (2013).

29. Pejler, G., Abrink, M., Ringvall, M. \& Wernersson, S. Mast cell proteases. Advances in immunology 95, 167-255, https://doi. org/10.1016/S0065-2776(07)95006-3 (2007).

30. Andersson, M. K., Karlson, U. \& Hellman, L. The extended cleavage specificity of the rodent beta-chymases rMCP-1 and mMCP-4 reveal major functional similarities to the human mast cell chymase. Molecular immunology 45, 766-775, https://doi.org/10.1016/j. molimm.2007.06.360 (2008).

31. Tchougounova, E., Pejler, G. \& Abrink, M. The chymase, mouse mast cell protease 4, constitutes the major chymotrypsin-like activity in peritoneum and ear tissue. A role for mouse mast cell protease 4 in thrombin regulation and fibronectin turnover. J Exp Med 198, 423-431, https://doi.org/10.1084/jem.20030671 (2003).

32. Desbiens, L. et al. Significant Contribution of Mouse Mast Cell Protease 4 in Early Phases of Experimental Autoimmune Encephalomyelitis. Mediators of inflammation 2016, 9797021, https://doi.org/10.1155/2016/9797021 (2016).

33. Tchougounova, E., Forsberg, E., Angelborg, G., Kjellen, L. \& Pejler, G. Altered processing of fibronectin in mice lacking heparin. a role for heparin-dependent mast cell chymase in fibronectin degradation. The Journal of biological chemistry 276, 3772-3777, https://doi.org/10.1074/jbc.M008434200 (2001).

34. Vangansewinkel, T. et al. Mast cells promote scar remodeling and functional recovery after spinal cord injury via mouse mast cell protease 6. FASEB journal: official publication of the Federation of American Societies for Experimental Biology 30, 2040-2057, https:// doi.org/10.1096/f.201500114R (2016).

35. Beghdadi, W. et al. Mast cell chymase protects against renal fibrosis in murine unilateral ureteral obstruction. Kidney Int 84, 317-326, https://doi.org/10.1038/ki.2013.98 (2013).

36. Hong, L. T. A. et al. An injectable hydrogel enhances tissue repair after spinal cord injury by promoting extracellular matrix remodeling. Nat Commun 8, 533, https://doi.org/10.1038/s41467-017-00583-8 (2017).

37. Klapka, N. et al. Suppression of fibrous scarring in spinal cord injury of rat promotes long-distance regeneration of corticospinal tract axons, rescue of primary motoneurons in somatosensory cortex and significant functional recovery. The European journal of neuroscience 22, 3047-3058, https://doi.org/10.1111/j.1460-9568.2005.04495.x (2005).

38. Zhu, Y. et al. Hematogenous macrophage depletion reduces the fibrotic scar and increases axonal growth after spinal cord injury. Neurobiology of disease 74, 114-125, https://doi.org/10.1016/j.nbd.2014.10.024 (2015).

39. Bradbury, E. J. et al. Chondroitinase ABC promotes functional recovery after spinal cord injury. Nature 416, 636-640, https://doi. org/10.1038/416636a (2002).

40. James, N. D. et al. Chondroitinase gene therapy improves upper limb function following cervical contusion injury. Experimental neurology 271, 131-135, https://doi.org/10.1016/j.expneurol.2015.05.022 (2015).

41. Renault-Mihara, F. et al. Beneficial compaction of spinal cord lesion by migrating astrocytes through glycogen synthase kinase-3 inhibition. EMBO Mol Med 3, 682-696, https://doi.org/10.1002/emmm.201100179 (2011).

42. Tchougounova, E. et al. A key role for mast cell chymase in the activation of pro-matrix metalloprotease-9 and pro-matrix metalloprotease-2. The Journal of biological chemistry 280, 9291-9296, https://doi.org/10.1074/jbc.M410396200 (2005).

43. Lundequist, A., Abrink, M. \& Pejler, G. Mast cell-dependent activation of pro matrix metalloprotease 2: A role for serglycin proteoglycan-dependent mast cell proteases. Biological chemistry 387, 1513-1519, https://doi.org/10.1515/BC.2006.189 (2006).

44. Suzuki, K., Lees, M., Newlands, G. F., Nagase, H. \& Woolley, D. E. Activation of precursors for matrix metalloproteinases 1 (interstitial collagenase) and 3 (stromelysin) by rat mast-cell proteinases I and II. Biochem J 305(Pt 1), 301-306 (1995).

45. Saunders, W. B., Bayless, K. J. \& Davis, G. E. MMP-1 activation by serine proteases and MMP-10 induces human capillary tubular network collapse and regression in 3D collagen matrices. J Cell Sci 118, 2325-2340, https://doi.org/10.1242/jcs.02360 (2005).

46. Johnson, J. L., Jackson, C. L., Angelini, G. D. \& George, S. J. Activation of matrix-degrading metalloproteinases by mast cell proteases in atherosclerotic plaques. Arterioscler Thromb Vasc Biol 18, 1707-1715 (1998).

47. Frank, B. T., Rossall, J. C., Caughey, G. H. \& Fang, K. C. Mast cell tissue inhibitor of metalloproteinase-1 is cleaved and inactivated extracellularly by alpha-chymase. J Immunol 166, 2783-2792 (2001).

48. Okada, S., Hara, M., Kobayakawa, K., Matsumoto, Y. \& Nakashima, Y. Astrocyte reactivity and astrogliosis after spinal cord injury. Neurosci Res, https://doi.org/10.1016/j.neures.2017.10.004 (2017).

49. Okada, S. et al. Blockade of interleukin-6 receptor suppresses reactive astrogliosis and ameliorates functional recovery in experimental spinal cord injury. J Neurosci Res 76, 265-276, https://doi.org/10.1002/inr.20044 (2004).

50. Raposo, C. \& Schwartz, M. Glial scar and immune cell involvement in tissue remodeling and repair following acute CNS injuries. Glia 62, 1895-1904, https://doi.org/10.1002/glia.22676 (2014).

51. Shechter, R., Raposo, C., London, A., Sagi, I. \& Schwartz, M. The glial scar-monocyte interplay: a pivotal resolution phase in spinal cord repair. PLoS One 6, e27969, https://doi.org/10.1371/journal.pone.0027969 (2011).

52. Funk, L. H., Hackett, A. R., Bunge, M. B. \& Lee, J. K. Tumor necrosis factor superfamily member APRIL contributes to fibrotic scar formation after spinal cord injury. J Neuroinflammation 13, 87, https://doi.org/10.1186/s12974-016-0552-4 (2016).

53. Sorokin, L. The impact of the extracellular matrix on inflammation. Nat Rev Immunol 10, 712-723, https://doi.org/10.1038/nri2852 (2010).

54. Bonnans, C., Chou, J. \& Werb, Z. Remodelling the extracellular matrix in development and disease. Nat Rev Mol Cell Biol 15, 786-801, https://doi.org/10.1038/nrm3904 (2014).

55. Dooley, D. et al. Cell-Based Delivery of Interleukin-13 Directs Alternative Activation of Macrophages Resulting in Improved Functional Outcome after Spinal Cord Injury. Stem Cell Reports 7, 1099-1115, https://doi.org/10.1016/j.stemcr.2016.11.005 (2016).

56. Basso, D. M. et al. Basso Mouse Scale for locomotion detects differences in recovery after spinal cord injury in five common mouse strains. J Neurotrauma 23, 635-659, https://doi.org/10.1089/neu.2006.23.635 (2006). 
57. Geurts, N. et al. Basophils are dispensable for the recovery of gross locomotion after spinal cord hemisection injury. J Leukoc Biol 99, 579-582, https://doi.org/10.1189/jlb.3AB0815-370R (2016).

58. Zhu, Y., Soderblom, C., Trojanowsky, M., Lee, D. H. \& Lee, J. K. Fibronectin Matrix Assembly after Spinal Cord Injury. J Neurotrauma 32, 1158-1167, https://doi.org/10.1089/neu.2014.3703 (2015).

\section{Acknowledgements}

The authors thank Leen Timmermans (Hasselt University) for her help with the qPCR analysis. This study was supported by grants from Fund for Scientific Research Flanders (FWO-Vlaanderen) to S.H. (G.0389.12, G0A5813) and N.G. (1.2.917.14N), and from 'Agency for Innovation by Science and Technology in Flanders' (IWT-Vlaanderen) to T.V. (101517) and S.L. (131230).

\section{Author Contributions}

T.V. \& S.L. both contributed to experimental design, data collection, data interpretation and manuscript writing. N.G. contributed to experimental design, data collection, data interpretation and revision of the manuscript. K.Q. contributed to data collection and revision of the manuscript. D.D. \& G.P. contributed to experimental design and revision of the manuscript. S.H. contributed to experimental design, data interpretation and reviewed the manuscript. All authors discussed the results and contributed significantly to the final version of the manuscript.

\section{Additional Information}

Supplementary information accompanies this paper at https://doi.org/10.1038/s41598-019-39551-1.

Competing Interests: The authors declare no competing interests.

Publisher's note: Springer Nature remains neutral with regard to jurisdictional claims in published maps and institutional affiliations.

(c) (i) Open Access This article is licensed under a Creative Commons Attribution 4.0 International License, which permits use, sharing, adaptation, distribution and reproduction in any medium or format, as long as you give appropriate credit to the original author(s) and the source, provide a link to the Creative Commons license, and indicate if changes were made. The images or other third party material in this article are included in the article's Creative Commons license, unless indicated otherwise in a credit line to the material. If material is not included in the article's Creative Commons license and your intended use is not permitted by statutory regulation or exceeds the permitted use, you will need to obtain permission directly from the copyright holder. To view a copy of this license, visit http://creativecommons.org/licenses/by/4.0/.

(c) The Author(s) 2019 